\title{
A WEB APP TO GENERATE AND DISSEMINATE NEW KNOWLEDGE ON URBAN GREEN SPACE QUALITIES AND THEIR ACCESSIBILITY
}

\author{
R. Hecht ${ }^{1}$ *, M. Artmann ${ }^{1}$, P. Brzoska ${ }^{1}$, D. Burghardt ${ }^{2}$, S. Cakir ${ }^{1}$, A. Dunkel ${ }^{2}$, M. Gröbe ${ }^{2}$, M. Gugulica ${ }^{2}$, K. Krellenberg ${ }^{1,3}$, \\ N. Kreutzarek ${ }^{4}$, S. Lautenbach ${ }^{5}$, C. Ludwig ${ }^{6}$, D. Lümkemann ${ }^{4}$, G. Meinel ${ }^{1}$, M. Schorcht ${ }^{1}$, A. Sonnenbichler ${ }^{4}$, C. Stanley ${ }^{1}$, \\ J. Tenikl ${ }^{7}$, M. Wurm ${ }^{7}$, A. Zipf ${ }^{5,6}$
}

${ }^{1}$ Leibniz Institute of Ecological Urban and Regional Development (IOER), Dresden, Germany

- (r.hecht, m.artmann, p.brzoska, s.cakir, g.meinel, m.schorcht, c.stanley)@ioer.de

${ }^{2}$ Institute of Cartography, Technische Universität Dresden, Dresden, Germany

- (dirk.burghardt, alexander.dunkel, mathias.groebe, madalina.gugulica)@tu-dresden.de

${ }^{3}$ University of Vienna, Department of Geography and Regional Research, Vienna, Austria - kerstin.krellenberg@univie.ac.at

${ }^{4}$ ISB AG, Karlsruhe, Germany - (niklas.kreutzarek, dennis.luemkemann, andreas.sonnenbichler)@isb-ag.de

${ }^{5}$ Heidelberg Institute for Geoinformation Technology (HeiGIT) gGmbH at Heidelberg University, Heidelberg, Germany - sven.lautenbach@heigit.org

${ }^{6}$ GIScience Research Group, Institute of Geography, Heidelberg University, Heidelberg, Germany - (christina.ludwig, zipf)@uni-heidelberg.de

${ }^{7}$ German Remote Sensing Data Center, German Aerospace Center (DLR), Weßling-Oberpfaffenhofen, Germany - (julia.tenikl, michael.wurm)@dlr.de

KEY WORDS: Urban Green Spaces, Web App, Dashboard, Data Integration, Location-Based Services, Smart Cities, Open Data

\begin{abstract}
:
Urban green spaces (UGSs) can provide important ecosystem services for citizens and their well-being. To make use of these services according to UGS user demands, urban residents, tourists, and city administrations should know where UGSs are located, what qualities they have and how to reach them on convenient routes. This paper presents a novel digital infrastructure which combines and fuses different data to map UGSs and their qualities, and makes this information available in a web app. The interactive information service of the app aims to support citizens to explore and search for suitable UGSs and to provide routing options to reach them based on their preferences. Via implicit and explicit feedback functions included in the app, further information on UGS users' preferences can be collected to enhance the overall knowledge basis, while respecting data privacy issues. The underlying data base consists primarily of open and volunteered geographic data, which allows for transferability to other cities. This paper describes the system design, its backend and front-end components, as well as the process of development and deployment of the system in two pilot cities. Preliminary results of the piloting in the two cities are presented, focusing on user preferences for UGSs searches. The added value of the web app for city residents and the role of the newly gained knowledge for urban planning is discussed and reflected upon.
\end{abstract}

\section{INTRODUCTION}

\subsection{Relevance of Urban Green Spaces}

To ensure good living conditions and well-being for urban residents, urban green spaces (UGSs), such as parks, gardens, flower strips, avenues or forests play an important role. UGSs provide a range of urban ecosystem services (UESS) and can thus make a significant contribution to the quality of life in cities (Bolund, Hunhammer, 1999; James et al., 2015). For example, UGSs can show positive effects on the urban climate, the health of the population, and on biodiversity. They can serve as places for experiencing nature, for relaxation and for social interactions (Gómez-Baggethun, Barton, 2013; Grunewald et al., 2018; Tost et al., 2019).

In consequence, the demand for UGSs by urban citizens is high. This underpins once again the recent lockdowns and measures related to the COVID-19 pandemic (Kleinschroth, Kowarik, 2020). Based on analyses of smartphone-based physical activity data in Oslo, a study revealed an increase in outdoor activity compared to the 3-year average for the same days of the year (Venter et al., 2020). In view of climate change and related heat events in cities, provision and accessibility of UGSs are of high importance. In this context, some studies highlight elderly and families with children as vulnerable groups that would profit most from UGSs (Grunewald et al., 2017; Kabisch, Krämer, 2020; Sikorska et al., 2020).

Hence, in the effort of liveable cities despite population growth and densification tendencies, the protection and evaluation of UGSs play an important role (Gómez-Baggethun, Barton, 2013; Haase et al., 2014). We argue that to strengthen a comprehensive assessment of UGS and to provide enhanced information to urban citizens and urban planning, it needs novel digital infrastructures that take user preferences into account.

\subsection{Lack of Knowledge about Urban Green Spaces}

Studies show that in addition to good accessibility, factors such as the size of UGSs or individual demands on their qualities and functions influence whether and how often public UGSs are visited (Krellenberg et al., 2021; Tost et al., 2019; Schipperijn et al., 2010). In order to allow urban residents to best benefit from the positive effects of UGSs, it is important to provide them with information where to find UGSs according to their preferences, how they are equipped, and how they can be reached. So far, such information hardly exists. Although parks or playgrounds can be

\footnotetext{
* Corresponding author
} 
found with common online mapping services, a search for UGSs based on specific features (e.g. trees, shady areas, playgrounds or public toilets) is to our knowledge currently not possible with any service. In order to develop such an information service, it requires comprehensive and, most importantly, accessible datasets, which are not available in sufficient quality for most cities and can be extremely heterogeneous in their data structure and semantics (Feltynowski et al., 2018). Although some local authorities offer their data openly, the datasets are often limited to designated parks and rarely include semi-public or informal UGSs, such as publicly accessible green spaces in cooperative apartment complexes or brownfields. Thus, for an equitable provision and accessibility of UGSs, urban planning should include precise information on the current city-wide supply of all publicly accessible UGSs as well as the actual demand on UGSs by citizens. In addition to the equipment and quality of UGSs, it is therefore of particular interest to know how individual green spaces are perceived and used by the people. In this way, deficits in the green space supply can be revealed.

\subsection{Benefits of Mobile Apps in Generating Knowledge}

Public participation geographic information systems (PPGIS) or participatory geographic information systems (PGIS) combine participatory methods with GIS to gather insights from the public to be used in the planning process (Brown, Kyttä, 2014). These approaches have already been utilised to support UESS assessments towards a better understanding of the use of urban green spaces (Rall et al., 2017, Heikinheimo et al., 2020). Developed nature-recreation apps, for example, aim at supporting GPS-tagged site identification and route mapping (Jepson, Ladle, 2015). Different apps already exist that show some similarities with the requirements described above, such as komoot, CityOases, CyberCardeto, DoPrírody, or outdooractive. The CyberCardeto app was developed to display green space qualities and to extract user data in terms of behaviour and movement patterns. People in Prague can use the DoPrírody app to find the most suitable and quickest reachable green space through navigation support (Ćábelka, Jakl, 2017). In the CityOases app (Banko et al, 2020), users can indicate which activities they undertake on which green spaces and rate them according to various criteria. This information is intended to support other users in their choice of green spaces as well as to help urban planners in the design of open spaces. Apps offer the advantage to use sensors and functionalities of the smartphone and allow direct access to the fed information (Hoffimann et al., 2018). However, the knowledge generated by all these apps is limited to individual green spaces. Thus, to actually support urban planning, the user evaluations would have to be combined with spatial aspects to enable an evaluation for all UGSs in a city.

\subsection{Aim}

The main aim of this paper is to present a novel digital infrastructure and a web app called meinGrün (myGreen) supporting citizens to perform recreational activities on the best suitable UGSs according to their demands and preferences. Since the web app is intended to be used primarily on mobile devices it represents a location-based service (LBS) supported by a novel routing service. The developed system architecture as well as initial results of piloting in two German cities, particularly Dresden and Heidelberg, are presented. The focus of the presented results lies on user and search statistics and a broader discussion of the relevance of the system for urban planning.

\section{INFRASTRUCTURE}

In the following, the general architecture of services and the individual components developed for the app are introduced. At first, the conceptual foundations necessary for content development are presented.

\subsection{Conceptual Basis and System Requirements}

The development of the app was based on the concept of UESS. Accordingly, UGSs are considered particularly important for providing cultural ecosystem services (CES) (Palliwoda et al., 2020), where the benefit occurs while perceiving and using UGSs (Buchel, Frantzeskaki, 2015). Tratalos et al. (2016) argue that CES can be measured, among others, by considering the quality of UGSs and the activities performed on it. This has been taken up for the app design, focusing on different activities. For the valuation of UGSs, both, the demand and the supply side of CES for performing certain activities are considered (Fig 1) (see also Beichler, 2015; Chen et al., 2020; Voigt et al., 2014). Supply includes the amenities and characteristics of UGSs, while demand is the requirements that users place on UGS amenities and characteristics. Information on the demand side was collected via surveys (Krellenberg et al., 2021), which is a common procedure for that purpose (e.g. Campbell et al., 2016; Fischer et al., 2018). This way the relevant criteria for the activities were determined.

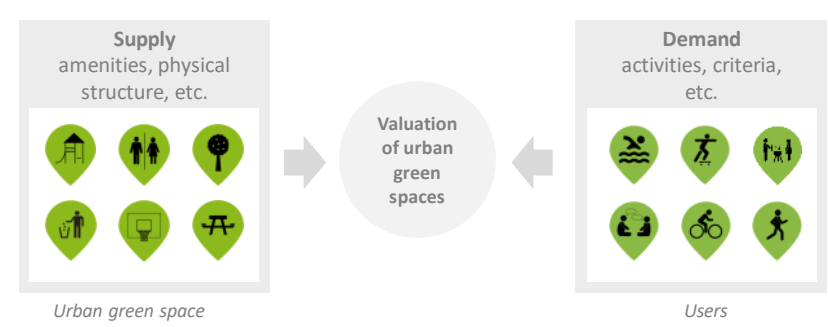

Figure 1. Valuation concept.

From the surveys, relevant activities, their associated criteria as well as their relative weights were derived. A multi-criteria evaluation approach (Krellenberg et al, 2021; Cakir, 2021) is then used to determine the suitability of UGS by indicators. Since the demand side is very subjective and can vary from user to user for the same activity (Massoni et al., 2018), a function is implemented in the app that allows to individually adjust the weights of the criteria per activity. To keep the app easy to use, only most important physical and passive recreational activities according to the survey results are listed. If a user prefers other or several activities, a search based on amenities and characteristics (summarized as 'criteria') can be conducted. Undecided users can simply explore all UGSs without any restrictions (see 2.2.1 for more details). In addition, the app displays amenities as points of interests (POIs). In fact, it was found that users accept longer travel times when green spaces are richly equipped (Zwierzchowska et al., 2018). Since the accessibility of UGSs significantly influences their frequency of use (Schipperijn et al., 2010), a routing function was developed that takes into account factors such as the presence of vegetation or noise in order to make visits to more distant UGSs more attractive (Wu et al., 2020). To promote sustainable mobility, routes are provided for pedestrians and cyclists only.

\subsection{System Architecture and Components}

The system design consists of two front-end components: the web/mobile app meinGrün and an associated browser-based dashboard for the administration, both communicating with 
backend services via HTTPS protocol (Fig. 2). The backend services run on bare metal and virtual servers in a distributed environment, each providing a specific function (data, processing services) used by the front-end components. An iterative approach was chosen for the app development, which allowed the functions to be constantly optimised. The involvement of beta testers from the public in the development process at an early stage enhanced the usability of the app (see 3.2).

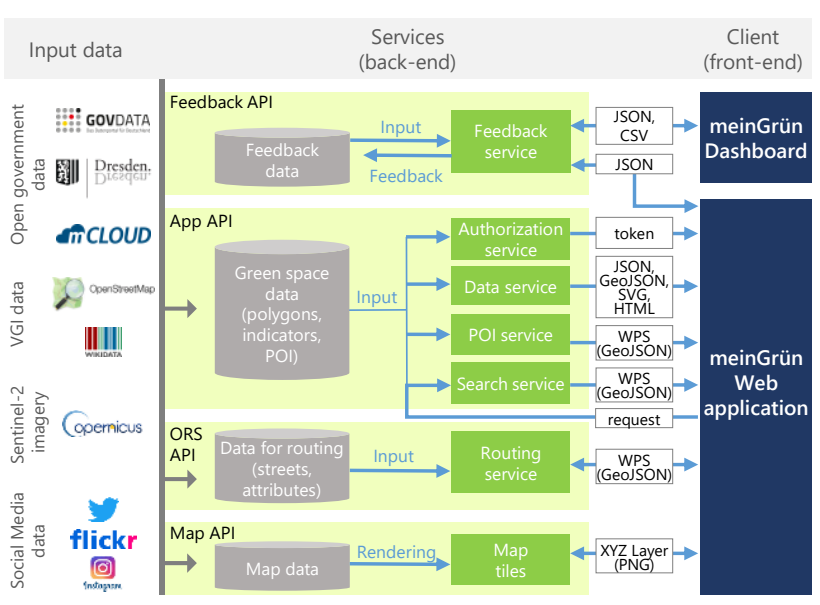

Figure 2. System design.

\subsubsection{Data and search services (App API)}

The meinGrün App API was developed to provide four different services that are authorization service, data service, POI service, and search service.

The authorization service is used for the generation and provision of a bearer token in order to prevent unauthorized access to the API.

The data service provides the data necessary for app usage, which are the green space polygons, POI icons and form data. The green space polygons are provided as GeoJSON objects. The POI icons for displaying the points of the POI service are provided in SVG format and serve to facilitate orientation. Search form data is provided as JSON, which contains all definitions for rendering the search dialog as well as the default parameters of the search service. Additionally, general information related to the app, such as terms of use, data sources, and imprint are provided as HTML as part of this service.

The POI service returns a GeoJSON feature collection containing available points for any selected green target. The service retrieves the POI geometries from the database. For the visualisation, the app accesses the necessary POI icons the data service on the client side.

The search service handles the search queries for finding suitable UGSs with three different search functions. All of them accept a search request containing relevant settings (e.g. search parameters, current map extent) and returning a GeoJSON as a response, including the geometry objects, as well as the distance to the user and a suitability score for each UGS:

- activity-search: This function returns the best UGS polygons (maximum of 20 in current map extent) that are particularly suitable for certain activities. A total of twenty different physical (e.g. jogging, Frisbee or table tennis) and passive recreational activities (e.g. reading, observing nature or sunbathing) are considered. The function accepts a search request including the map extent, selected activity as well as the default or possibly modified weights of criteria associated with the selected activity. The score produced for each green space shows how suitable it is to pursue the selected recreational activity. The score calculation follows a multi-criteria decision making (MCDM), which is described in more detail in Cakir et al. (2021).

- criteria-search: The in- and outputs of this function are similar to the activity-search function as the score calculation also follows the MCDM approach. The score indicates how well the UGSs meets one or more selected criteria, with a total of 36 criteria to choose from. The criteria can be combined and weighted as desired.

- fast-search: The fast search allows to search via a free text, such as activities, UGSs criteria or place names. By means of an auto-completion based on a defined dictionary, a hit list is returned after each input of a character. A search for 'gr', for example, would return 'green' or the place name of the park 'Großer Garten' in the hit list.

\subsubsection{Routing service (ORS API)}

The routing service of the web app meinGrün is built on the OpenRouteService (ORS), an open source routing engine based on freely available, user generated data of the OpenStreetMap (OSM) project (Neis, Zipf 2008; Lautenbach et al., 2020). The ORS software is written in Java and provides its services via an HTTPS interface served by Tomcat. Its source code is openly available on GitHub (GIScience Research Group \& HeiGIT, 2021) if one wants to set-up an own ORS instance. The official ORS instance can be queried globally through its public API (OpenRouteService, 2021). The ORS API provides different spatial services such as routing requests or isochrones analyses. The ORS routing service offers different routing profiles such as car, bike or pedestrian as well as several other query attributes (e.g. avoid ferries or toll ways) to adjust the generated route to the user's needs. Moreover, it allows for the integration of additional data in the route calculation such as traffic noise levels to generate quiet routes or vegetation data to generate green routes for pedestrians and cyclists (Novack et al., 2018; Wang et al., 2020). The calculation of the shortest route between two points is based on a weighted graph whose costs depend on the street length. To generate for example a green route, these costs are increased for streets that contain little vegetation. For details on the weighting please refer to Ludwig et al. (accepted). Based on such weighted graphs, an optimal route can be calculated using different algorithms such as the common Dijkstra's algorithm or more efficient ones such as the A* algorithm (Zeng, Church, 2009). Here, a new instance of the ORS was created which extends the original ORS by implementing shaded routes for pedestrians and cyclists and integrating more accurate region specific data basis for generating green routes. Depending on the regional data availability, the required data to calculate green or shaded routes was derived from $3 \mathrm{D}$ point cloud data, municipal tree cadastre data or openly available OSM and Sentinel-2 satellite imagery (Hecht et al., 2008; Ludwig et al., 2021).

\subsubsection{Feedback service (Feedback API)}

The feedback system is used to collect knowledge from the users within the meinGrün app environment, to store it in a database and to reuse it by making it available in the dashboard. Knowledge production can take place in two ways: 1 . Knowledge is generated via collected implicit feedback through the use of the app, and without the user being aware of this. 2. The user can actively provide knowledge via explicit feedback. The implicit user feedback is collected via an event tracker during app usage. Events consist of a name and optional data. Tracked events in the app are the installation of the app to the home screen, the time the user interface took to load, the city the app was used for, the selection of a search option, and the search itself. Explicit 
feedback is collected via survey dialogs, which consist of one or more individual questions. The survey dialogs support closeended questions with a scale and open-ended questions.

To associate explicit and implicit feedback with a particular user, a unique identifier gets send with the feedback data. The identifier is a randomly generated string that is created on the first feedback response by the feedback service and stored in the browser cookies for every subsequent response. In addition, each response is marked with the date and time it was received.

\subsubsection{Base map service (Map API)}

A base map was developed for the application, which includes essential reference objects for user orientation. The great advantage of an own map service is the complete control over all map features and their visualization. Thereby, it is possible to optimally match the map and app design and create recognizability value. The map is hosted and loaded as raster tiles by the app on demand via a URL template with the variables $\mathrm{XYZ}$ (rows, columns, and zoom levels of the map tiles as raster graphics). The map tiles are cached on the application's server to allow faster access. For performance reasons, hosting on an own server is the most reliable source. For this, a Tileserver GL was used with the caching function of the Apache web server, serving the map from pre-rendered vector tiles stored in the MBTiles format. Then tiles get converted into raster graphics according to a predefined map style. For further details about the generation of the map, see Gröbe et al. (2020). Instead of the own produced tile set, other providers like OpenMapTiles or Mapbox can be used.

\subsubsection{Front-end web app}

The web app meinGrün was implemented as a progressive web app (PWA), a unifying technology for web apps and native apps (Biørn-Hansen et al., 2017) to reach a wide range of users. The responsiveness and platform independence of the app leads to an easy access on PCs, tablets, and smartphones via the browser engine. This gives users the option of exploring the app at home or using it spontaneously when outside on their mobile phone.

Being a PWA, the app is stored on the user's device or browser for fast start-up and an icon can be placed on the home screen for easy access. Upon start, the app retrieves the latest search form definitions and shows a map centred on the user's position. The user can explore the map and UGSs visually, or use the search function (see 2.2.1). A fast search can be started by entering a keyword into the search field (activity, criteria, place name). Alternatively, a detailed search by individual activities or search criteria, such as facilities (benches, restrooms, basketball court, skate park), natural elements (trees, water elements), or other specific characteristics (area size, flat meadow, greenness, shading), can be selected. The offered criteria are the top criteria resulting of the study of users' preferences (Krellenberg et al., 2021). The user can also select on the importance of certain criteria, e.g. a meadow when searching for a place to play soccer. When the user submits the search, its current position or, if not activated, the coordinates of the map centre is added to the search request and sent to the search service. The search results are shown as green polygons on the map and as a sortable hit list ('Trefferliste') of UGSs including their score ('Eignung'), name and distance ('Entfernung'), which the user can toggle between (Fig. 3a, b). The selection of a UGS in the list highlights its polygon on the map. Its selection on the map displays the associated POIs with an info box showing its name, category and score (Fig. 3c). From here, users can request routing options from their current positions and preferences of either walking or cycling. This request is then sent to the routing service backend.
As a result, different routes are shown on the map, such as the greenest ('Grün'), quietest ('Leise'), shadiest ('Schattig') and fastest ('Schnell') routes to the selected UGS along with their distance and estimated travel time (Fig. 3d).

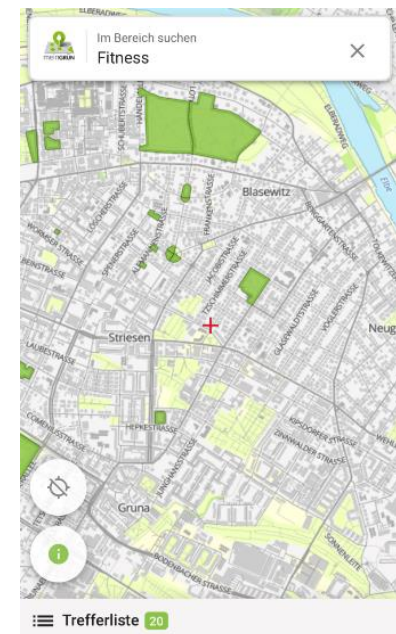

a)

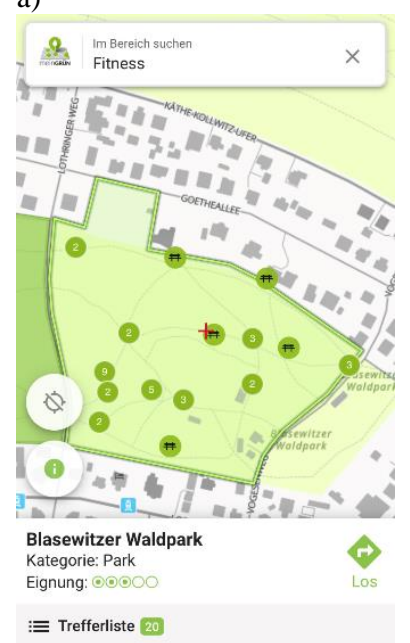

c)

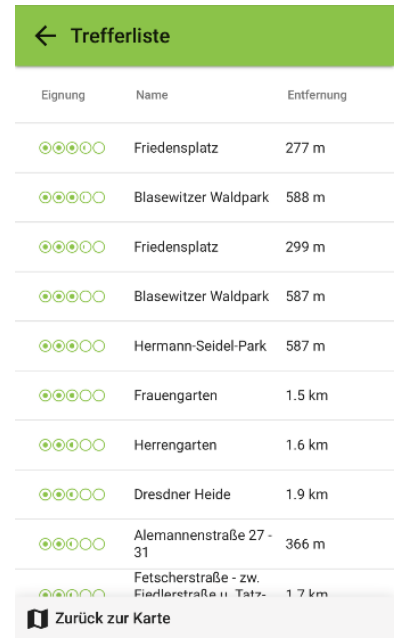

b)

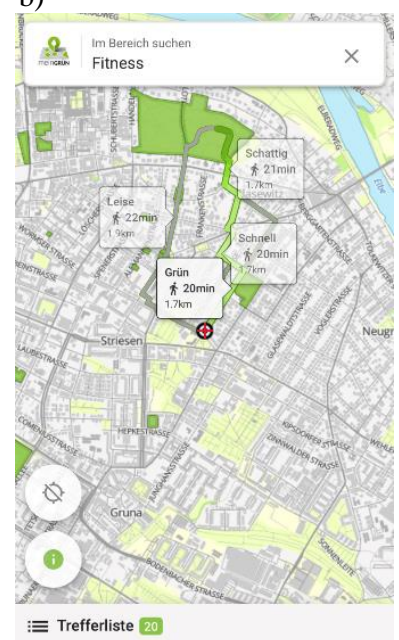

d)

Figure 3. Screenshots of the web app meinGrün in mobile use after querying the term 'fitness' for the city of Dresden: a) map of appropriate UGSs in the surrounding of the app user (20 best), b) list view of the UGSs, c) detailed view of a selected UGS, d) routing options after selecting the walking mode

\subsubsection{Front-end dashboard}

The meinGrün dashboard is a web-based user interface that provides a simple way to see and manage implicit and explicit feedback. Features include creating and editing surveys for explicit feedback, viewing individual survey responses, as well as displaying a summary of the responses and the export of the implicit feedback data as a CSV file. This makes it possible to easily extract the user and search statistics.

\subsection{Data Basis and Processing}

\subsubsection{Polygon generation}

The polygon structure as a spatial reference unit for the calculation of indicators and for the definition of green space targets in the web app required the development of a method to automatically generate meaningful target polygons based on 
physical barriers, the greenness as well as their public accessibility. The barriers were created from the street network as well as boundaries of certain adjacent land use class combinations. The approach based on OSM and Sentinel-2 data is described in Ludwig et al. (2021). In the work here, official city block data and green space polygon data were additionally used to further support the polygon generation process.

\subsubsection{Indicator calculation}

The indicators for assessing the suitability of UGSs based on criteria have been described by Krellenberg et al. (2021). The 36 criteria and the associated indicators are divided into three categories:

- Physical structure: All indicators that describe the biophysical green structure of the biotic and abiotic elements, i.e. the share of a certain vegetation types (trees, meadow) in the UGSs in percent, the urban green volume per area $\left(\mathrm{m}^{3} / \mathrm{m}^{2}\right)$ or the species diversity measured by an adjusted Shannon index (Fischer, Mölder, 2017).

- Amenities: The density of facilities that support recreation, such as the number of benches per hectare or the presence of certain features such as playgrounds, basketball court, soccer goals, or barbeque places.

- Perceived qualities: UGSs qualities perceived through human-environmental interaction, such as silence, aesthetics, naturalness or cleanliness.

Various data sources were used for the indicator calculation. For physical structure indicators, data from the Sentinel-2 mission of the European Copernicus Program were used to derive the vegetation cover as well as the proportion of different vegetation types with a spatial resolution of 10x10m (Krüger et al., 2018; Weigand et al., 2020). Alternatively, aerial images and LiDAR data can be used if available for the city for processing urban green volume (Hecht et al., 2008). OSM data play a major role as a basis for indicators to describe amenities. However, there is also an increasing amount of open municipal data available that contains information on infrastructure (e.g. playgrounds, benches or toilets). These can also be used and fused with OSM data, whereby aspects of data quality aspects, incompatibilities regarding semantics and licensing rights must be taken into account. When it comes to quantifying perceived qualities of UGSs such as aesthetics, publicly available and geo-located social media data from Flickr, Instagram and Twitter were primarily used by calculating various metrics, such as user counts, photo/post counts or user days (as the cumulative count of users having visited particular UGSs on consecutive days). These metrics derived by using the privacy-aware abstraction routine for quantitative analysis from Dunkel et al. (2020) give further insights on how the public perceives and interacts with UGSs. Further details on the indicator calculation can be found in Krellenberg et al. (2021).

\subsubsection{POI data}

In addition, point data is visualized to make specific equipment on the green spaces localizable and to facilitate orientation. For this purpose, various POI data were collected, integrated and made available in the app. In total, 20 different POIs themes are offered, which are grouped into amenities related to activities: sports amenities (e.g. table tennis tables or soccer fields), recreation-related amenities (e.g. benches or sights) and amenities related to consumption (e.g. restaurants or supermarkets). As basis for the POIs mainly OSM, but also partly open data of the cities are used.

\section{PILOTING OF THE APP}

\subsection{Pilot Cities}

The app was developed for two pilot cities, Dresden and Heidelberg in Germany. Dresden is the capital city of the federal state Saxony with a population of 561,358 inhabitants (Landeshauptstadt Dresden, 2020). With a city size of 328.46 $\mathrm{km}^{2}$, Dresden's UGSs supply is about $101.8 \mathrm{~m}^{2}$ per capita (IOER, 2021). In contrast, Heidelberg being located in the federal state Baden-Württemberg, has a population of 149,722 inhabitants and a city size of about $108.83 \mathrm{~km}^{2}$ (Stadt Heidelberg, 2018). The UGSs supply per inhabitant is $91.1 \mathrm{~m}^{2}$ (IOER, 2021).

\subsection{Participatory Requirement Analysis and Beta-testing}

The app was developed by means of a user-centred design in which potential users were involved early and throughout the development process to ensure user satisfaction (Krellenberg et al., 2021). Participants were recruited through various formats (social media, flyer, press releases and advertising at local events) in the two pilot cities and beyond. In addition to the surveys conducted as part of the requirement analysis, users were involved in the beta testing of the app, which took place in the form of local events co-promoted by the pilot cities. In a first beta testing event, volunteers $(n=28)$ tested the usability and user experience especially on effectiveness, efficiency and satisfaction with the search and routing functions. This involved the 'Think Aloud' method (van Someren et al., 1994) for some beta testers $(n=15)$. The procedure resulted in a total of 115 user comments and recommendations, with 70 of these implemented to optimize the user interface. The second beta test focused primarily on evaluating the proposed results from the web app, performed by an online questionnaire. Participants $(n=12)$ were asked to search for given activities as well as criteria of UGSs and to assess the results from the web app from 'very suitable' to 'not suitable'. The evaluations were then used to improve the indicator calculation before the upcoming launch of the app. Finally, implicit feedback was used to explore usability. For example, in the beta testing phase, implicit feedback was used to optimise the GUI for the search form, as it was found that simple search forms were preferred by users.

\subsection{App Launch}

The meinGrün app was launched in June 2020. Due to the COVID-19 pandemic, the launch took place entirely online at https://meingruen.org/. Virtual scavenger hunts were offered, where citizens could playfully explore the various features of the app on foot or by bike. The launch was backed by press releases and target group-specific social media campaigns (Twitter, Facebook, Instagram) with the support of the city administrations and thus attracted print, online and TV media attention.

\section{RESULTS}

\subsection{General User Statistics}

To be able to reveal the number of users over time, such as the number of unique users per week, implicit feedback data of the dashboard was used. According to this data, the app has been used by an average of 100 users per week since its launch, of which about $70 \%$ are using the app for the first time.

Since the launch, the app was used the most in the first week (453 users in total), of whom 365 were new and 88 returning users (Fig. 4). Until the $42^{\text {nd }}$ week of 2020 , the number of weekly users showed a fluctuating tendency. A larger decline, probably due to seasonal effects, was observed towards the end of 2020. A similar 
pattern of decline, albeit at an even lower absolute level, can be seen in the user statistics of the Vienna CityOases app (Banko et al., 2020). However, social media campaigns at the beginning of 2021 increased the number of weekly users again.

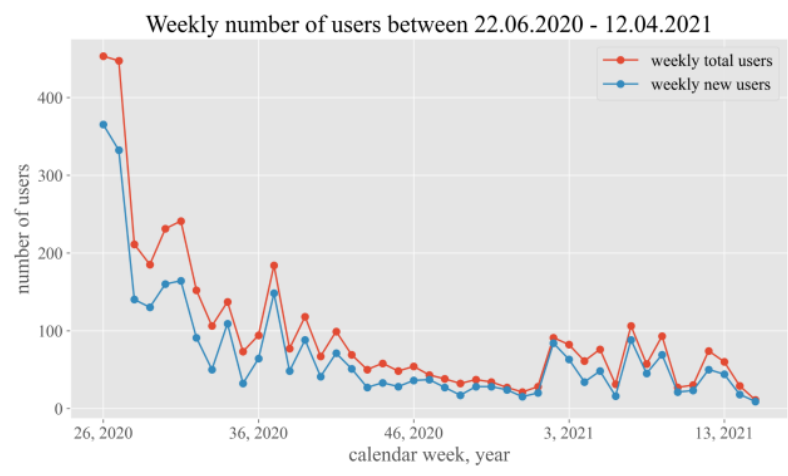

Figure 4. Weekly number of users who have used the app at least one day a week total users (weekly total users, red) and new users who have used the app for the first time in the respective week (weekly new users, blue).

\subsection{Search Statistics}

The analysis of the search statistics considered a much shorter period between 16.09 .2020 and 06.01 .2021 , as only since 16.09.2020 an implicit feedback function recorded the corresponding values. During this period, 718 searches were made by 263 users. In more than $40 \%$ of the cases $(n=294)$, a specific activity was searched for, in $35 \%$ of the cases $(n=248)$, one or more criteria were searched for, and in just under a quarter $(n=176)$, users have searched for a term using the fast search function.
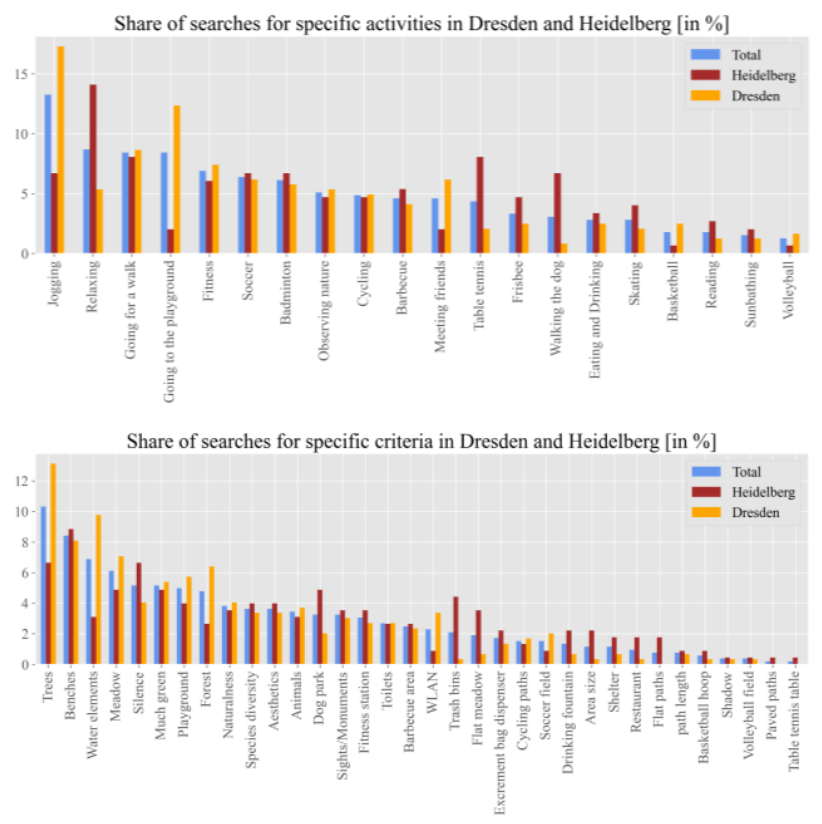

Figure 5. Search statistics: Share of searches for specific activities (top) and criteria in total (bottom), in Heidelberg and in Dresden.

Figure 5 shows the share of searches for specific activities and criteria in percent for all searches, as well as differentiated by the two cities of Dresden and Heidelberg. The most searched activity is jogging with a share of $13.3 \%$ and the least searched is volleyball with a share of $1.3 \%$. The activities jogging $(17.3 \%)$, going to the playground (12.3\%) and meeting friends (6.2\%) are the top searched activities in Dresden, whereas in Heidelberg the activities relaxing $(14.1 \%)$, table tennis $(8.1 \%)$ and walking the $\operatorname{dog}(6.7 \%)$ were the top three. The most frequently searched criterion is trees $(10.3 \%)$ and the least frequently table tennis tables and paved paths (both $0.2 \%$ ). In Dresden the criteria trees $(13.1 \%)$, water elements $(9.8 \%)$ and forest $(6.4 \%)$ are searched most often. In Heidelberg it was silence (6.6\%), dog park (4.9\%) and trash bins $(4.4 \%)$.

\section{DISCUSSION AND OUTLOOK}

It was shown that by combining user preferences and an indicator-based assessment approach based on various geospatial data, new knowledge about qualities of UGSs as well as the routes to them can be generated and interactively be disseminated via the web app. The evaluations of the search queries showed different patterns with regard to the activities and criteria in Dresden and Heidelberg. In the future, these statistics could be further spatially located in the future (e.g. at the level of city districts), in order to support spatially detailed deficit analyses with regard to the UGS supply. This knowledge can be used by UGS planners, for example to optimize green spaces according to citizen preferences. As the financial budget for the management and maintenance of UGSs is often limited, determining the value of each UGS can support the allocation of funds appropriately for future development and upgrading UGS according to the needs of the citizens. In this regard, spatial information provided by the user statistics can support urban planning to identify gaps between the supply of and demand on UESS, and to secure equal access to UGS for recreation activities (e.g. Suárez et al., 2020). With reference to routing service, the data can also be used to assess the attractiveness of cycling infrastructure in cities and thus support sustainable transport planning.

The app was tested under real conditions in the pilot cities. However, a challenge during the pilot period were the restrictions in connection with the containment of the COVID-19 pandemic thus potentially new preferences of urban residents. Some activities were not allowed due to physical distancing and the demand for unpopular, uncrowded green spaces may have increased. The almost a yearlong recorded user statistics indicate that the app has the potential to reach more users if it is promoted continuously (and not just at the beginning) through social media campaigns. Even though the number of users and searches per week already tells a lot about the usage of the app, there is even more potential to capture and evaluate other metrics, such as the number of returning users or the churn rate after first usage. In this regard, further research is needed to investigate the motivations of app users, in order to better promote the app to the relevant target groups and thus achieve more users and a longterm use of the app. This also includes more intensive use of the feedback service to query user satisfaction with the proposed UGSs or routes via the explicit feedback function.

The app also has transformative potential. The new information on attractive walking or cycling routes to them could create incentives to make everyday mobility more sustainable when visiting UGSs. At the same time, the app can support health and well-being of the users, by promoting forms of physical and passive recreation (exercising, closeness to nature, relaxing).

Overall, the app and the infrastructure of the services form a broad basis for further developments as well as scientific analyses. Transfer to comparable cities is an option, which might set the ground for a comparison of UGSs and UESS provided across cities. The use of free and globally available OSM, 
Sentinel-2 and social media data ensures good transferability to other cities and countries in the world where urban green space provision is especially poor and the demand for an app like the one proposed here is even greater.

\section{ACKNOWLEDGEMENTS}

The research is supported by the German Federal Ministry of Transport and Digital Infrastructure (BMVI) under the frame of mFUND, a research initiative funding $R \& D$ projects related to digital data-based applications for Mobility 4.0 (grant number 19F2073A). We would like to thank the administrations and citizens of the pilot cities for their support.

\section{REFERENCES}

Banko, G., Birli, B., Stickler, T., Sturn, T., Wannemacher, K., Wildenberg, M., Moorthy, I., 2020: Exploring and promoting green urban spaces in Vienna - can data about public perception help drive change. Data Collection. https://zenodo.org/record/3695015 (26 April 2021).

Beichler, S.A., 2015: Exploring the link between supply and demand of cultural ecosystem services - towards an integrated vulnerability assessment. Int. J. Biodivers. Sci. Ecosyst. Serv. Manag. 11(3), 250-263.

Biørn-Hansen, A., Majchrzak, T., Grønli, T-M., 2017: Progressive Web Apps: The Possible Web-native Unifier for Mobile Development. In Proc. of the 13th International Conference on Web Information Systems and Technologies WEBIST, 344-351. https://doi.org/10.5220/0006353703440351.

Bolund, P., Hunhammer, S., 1999: Ecosystem Services in Urban Areas. Ecol. Econ. 29, 293-301.

Brown, G., Kyttä, M., 2014: Key issues and research priorities for public participation GIS (PPGIS): A synthesis based on empirical research. Appl. Geogr. 46, 122-136.

Buchel, S., Frantzeskaki, N., 2015: Citizens' voice: A case study about perceived ecosystem services by urban park users in Rotterdam, the Netherlands. Ecosyst. Serv. 12, 169-177.

Čábelka, M., Jakl, M., 2017: DoPřírody! - geoinformation mobile application. AUC GEOGRAPHICA 52 (2), 249-257.

Cakir, S., Hecht, R., Krellenberg, K., 2021: Sensitivity analysis in multi-criteria evaluation of the suitability of urban green spaces for recreational activities. AGILE GIScience Ser., 2, 22, https://doi.org/10.5194/agile-giss-2-22-2021.

Campbell, L.K., Svendsen, E.S., Sonti, N.F., Johnson, M.L., 2016: A social assessment of urban parkland: Analyzing park use and meaning to inform management and resilience planning. Environ. Sci. Policy 62, 34-44.

Chen, S., Wang, Y., Ni, Z., Zhang, X., Xia, B., 2020: Benefits of the ecosystem services provided by urban green infrastructures: Differences between perception and measurements. Urban For. Urban Green. 54, 126774.

Dunkel, A., Löchner, M., Burghardt, D., 2020: Privacy-aware visualization of volunteered geographic information (VGI) to analyze spatial activity: a benchmark implementation. ISPRS Int. J. Geo-Inf. 9(10), 607 .
Feltynowski, M., Kronenberg, J., Bergier, T., Kabisch, N., Łaszkiewicz, E., Strohbach, M.W., 2018: Challenges of urban green space management in the face of using inadequate data. Urban For. Urban Green. 31, 56-66.

Fischer, C., Mölder, A., 2017: Trend to increasing structural diversity in German forests: results from National Forest Inventories 2002 and 2012. Ann. For. Sci. 74(4), 80.

Fischer, L.K., Honold, J., Botzat, A., Brinkmeyer, D., Cvejić, R., Delshammar, T., Elands, B., Haase, D., Kabisch, N., Karle, S.J., Lafortezza, R., Nastran, M., Nielsen, A.B., van der Jagt, A.P., Vierikko, K., Kowarik, I., 2018: Recreational ecosystem services in European cities: Sociocultural and geographical contexts matter for park use. Ecosyst. Serv. 31, 455-467.

GIScience Research Group \& HeiGIT, 2021. OpenRouteService (ORS). https://github.com/GIScience/openrouteservice (26 March 2020).

Gómez-Baggethun, E., Barton, D.N., 2013: Classifying and Valuing Ecosystem Services for Urban Planning. Ecol. Econ. 86, 235-245.

Gröbe, M., Dunkel, A., Burghardt, D., 2020: A new web map for the MeinGrün app, Abstr. Int. Cartogr. Assoc. 2, 8.

Grunewald, K., Li, J., Xie, G., Kümper-Schlake, L. (Hrsg.): Towards green cities: Urban biodiversity and ecosystem services in China and Germany. Cham: Springer International Publishing, 2018.

Grunewald, K., Richter, B., Meinel, G., Herold, H., Syrbe, R.-U., 2017: Proposal of indicators regarding the provision and accessibility of green spaces for assessing the ecosystem service "recreation in the city" in Germany. Int. J. Biodivers. Sci. Ecosyst. Serv. Manag 13 (2), 26-39.

Haase, D., Fantzeskaki, N., Elmqvist, T., 2014: Ecosystem Services in Urban Landscapes: Practical Applications and Governance Implications. Ambio 43, 407-412.

Hecht, R., Meinel, G., Buchroithner, M.F., 2008: Estimation of urban green volume based on single-pulse LiDAR data. IEEE Trans. Geosci. Remote Sens. 46(11), 3832-3840.

Heikinheimo, V., Tenkanen, H., Bergroth, C., Järv, O., Hiippala, T. Toivonen, T., 2020. Understanding the Use of Urban Green Spaces from User-generated Geographic Information. Landsc. Urban Plan. 201, 103845.

Hoffimann, E., Campelo, D., Hooper, P., Barros, H., Ribeiro, A. I., 2018: Development of a smartphone app to evaluate the quality of public open space for physical activity. An instrument for health researchers and urban planners. Landsc. Urban Plan. 177, 191-195.

IOER, 2021. Monitor of Settlement and Open Space Development; editing status 2021-03-17; re3data.org - Registry of Research Data Repositories, https://doi.org/10.17616/R3QF5P.

James, P., Banay, R.F., Hart, J.E., Laden, F., 2015: A Review of the Health Benefits of Greenness. Curr. Epidemiol. Rep. 2, 131142 
Jepson, P., Ladle, R. J. 2015: Nature apps: Waiting for the revolution. Ambio 44(8), 827-832.

Kabisch, N., Kraemer, R., 2020: Physical activity patterns in two differently characterized urban parks under conditions of summer heat. Environ. Sci. Policy 107, 56-65.

Kleinschroth, F., Kowarik, I., 2020: COVID-19 crisis demonstrates the urgent need for urban greenspaces. Front. Ecol. Environ. 18(6), 318-319.

Krellenberg, K., Artmann, M., Stanley, C., Hecht, R., 2021: What to do in, and what to expect from, urban green spaces - Indicatorbased approach to assess cultural ecosystem services. Urban For. Urban Green. 59, 126986.

Krüger, T., Hecht, R., Herbrich, J., Behnisch, M., Oczipka, M., 2018: Investigating the suitability of Sentinel-2 data to derive the urban vegetation structure. Proc. SPIE 10793. Remote Sensing Technologies and Applications in Urban Environments III, https://doi.org/10.1117/12.2325337.

Landeshauptstadt Dresden, 2020. Dresden in Zahlen: III. Quartal 2019. https://www.dresden.de/media/pdf/statistik/Dresden in_Zahlen_2019_III_Quartal.pdf (20 December 2020).

Lautenbach, S., Ludwig, C., Fendrich, S., Novack, T., Marx, S., Oleś, A., Zipf, A., 2020: "Optimal ans Ziel: Routing-Dienste auf Basis nutzergenerierter Geodaten - Herausforderungen und Lösungsansätze für globale Datensätze" In: Zagel, B., Loidl, M. (eds.) Geo-IT in Mobilität und Verkehr: Geoinformatik als Grundlage für moderne Verkehrsplanung und Mobilitätsmanagement, 89 - 108. Wichmann, Berlin.

Ludwig, C., Hecht, R., Lautenbach, S., Schorcht, M., Zipf, A., 2021: Mapping Public Urban Green Spaces Based on OpenStreetMap and Sentinel-2 Imagery Using Belief Functions, ISPRS Int. J. Geo-Inf. 10, 251.

Ludwig, C., Zipf, A., Lautenbach S., 2021: Assessment of green routes at the example of Heidelberg and Dresden, GIScience 2021, September 2021, Poznań, Poland (accepted paper).

Massoni, E.S., Barton, D.N., Rusch, G.M., Gundersen, V., 2018: Bigger, more diverse and better? Mapping structural diversity and its recreational value in urban green spaces. Ecosyst. Serv. $31,502-516$.

Neis, P., Zipf, A., 2008: OpenRouteService.org is three times "Open": Combining OpenSource, OpenLS and OpenStreetMaps. GIS Research UK (GISRUK 08). Manchester, UK.

Novack, T., Wang, Z., Zipf, A., 2018: A System for Generating Customized Pleasant Pedestrian Routes Based on OpenStreetMap Data. Sensors 18, 3794.

OpenRouteService. 2021. https://maps.openrouteservice.org (26 March 2020).

Palliwoda, J., Banzhaf, E., Priess, J.A., 2020: How do the green components of urban green infrastructure influence the use of ecosystem services? Examples from Leipzig, Germany. Landsc. Ecol. 35, 1127-1142.

Rall, E., Bieling, C., Zytynska, S., Haase, D., 2017. Exploring city-wide patterns of cultural ecosystem service perceptions and use. Ecol. Indic. 77, 80-95.
Schipperijn, J., Stigsdotter, U.K., Randrup, T.B., Troelsen, J., 2010: Influences on the use of urban green space - A case study in Odense, Denmark. Urban For. Urban Green. 9, 25-32.

Sikorska, D., Łaszkiewicz, E., Krauze, K., Sikorski, P., 2020: The role of informal green spaces in reducing inequalities in urban green space availability to children and seniors. Environ. Sci. Policy 108, 144-154.

Stadt Heidelberg, 2018: Amt für Stadtentwicklung und Statistik, Heidelberg, 2018. Heidelberger Datenatlas. http://ww2.heidelberg.de/datenatlas/ (29 March 2020).

Suárez, M., Barton, D.N., Cimburova, Z., Rusch, G.M., GómezBaggethun, E., Onaindia, M., 2020: Environmental Justice and Outdoor Recreation Opportunities: A Spatially Explicit Assessment in Oslo Metropolitan Area, Norway. Environ. Sci. Policy, 108, 133-143.

Tost, H., Reichert, M., Braun, U., Reinhard, I., Peters, R., Lautenbach, S., Hoell, A., Schwarz, E., Ebner-Priemer, U., Zipf, A., \& Meyer-Lindenberg, A., 2019: Neural correlates of individual differences in affective benefit of real-life urban green space exposure. Nat. Neurosci. 22, 1389-1393.

Tratalos, J. A., Haines-Young, R., Potschin, M., Fish, R., Church, A., 2016: Cultural ecosystem services in the UK: Lessons on designing indicators to inform management and policy. Ecol. Indic. 61, 63-73.

van Someren, M.W., Barnard, Y.F., Sandberg, J.A.C., 1994: The Think-Aloud Method: A Practical Guide to Modelling Cognitive Processes. Academic Press, San Diego.

Venter, Z., Barton, D., Gundersen, V., Figari, H., Nowell, M., 2020: Urban nature in a time of crisis: Recreational use of green space increases during the COVID-19 out-break in Oslo, Norway. Environ. Res. Lett. 15, 104075.

Voigt, A., Kabisch, N., Wurster, D., Haase, D., Breuste, J., 2014: Structural Diversity: A Multi-dimensional Approach to Assess Recreational Services in Urban Parks. Ambio 43(4), 480-491.

Wang, Z., Novack, T., Yan, Y., Zipf, A., 2020: Quiet Route Planning for Pedestrians in Traffic Noise Polluted Environments IEEE Trans. Geosci. Remote Sens. https://doi.org/10.1109/TITS.2020.3004660

Weigand, M., Staab, J., Wurm, M., Taubenböck, H., 2020: Spatial and semantic effects of LUCAS samples on fully automated land use/land cover classification in high-resolution Sentinel-2 data. Int. J. Appl. Earth Obs. Geoinf. 88, 102065.

Wu, J., Wang, B., Ta, N., Zhou, K., Chai, Y., 2020: Does street greenery always promote active travel? Evidence from Beijing. Urban For. Urban Green. 56, 126886.

Zeng, W., Church, R. L., 2009: Finding shortest paths on real road networks: the case for A*. Int. J. Geogr. Inf. Sci. 23(4), 531543.

Zwierzchowska, I., Hof, A., Iojă, I.-C., Mueller, C., Poniży, L., Breuste, J., Mizgajski, A., 2018: Multi-scale assessment of cultural ecosystem services of parks in Central European cities. Urban For. Urban Green. 30, 84-97. 